\title{
Pneumonia Detection using X-Ray Images with Deep Learning
}

\author{
Hanumant Magar \\ MIT College of \\ Engineering, Kothrud, \\ Pune , India
}

\author{
Sanket J Patil \\ MIT College of \\ Engineering, Kothrud, \\ Pune, India
}

\author{
Sahil R Waykole \\ MIT College of \\ Engineering, Kothrud, \\ Pune, India
}

\author{
Satyam D Sandikar \\ MIT College of Engineering, Kothrud, \\ Pune, India
}

\author{
Nikhil D Parakh \\ MIT College of Engineering, Kothrud, \\ Pune, India
}

\begin{abstract}
This study proposes a Convolutional neural network model trained from scratch to classify and detect the presence of pneumonia from a collection of chest X-ray image samples. Unlike other methods that rely solely on transfer learning approaches or traditional handcrafted techniques to achieve a remarkable classification performance, we constructed a Convolutional neural network model from scratch to extract features from a given chest $\mathrm{X}$ ray image and classify it to determine if a person is infected with pneumonia. This model could help mitigate the reliability and interpretability challenges often faced when dealing with medical imagery. Unlike other deep learning classification tasks with sufficient image repository, it is difficult to obtain a large amount of pneumonia dataset for this classification task; therefore, we deployed several data augmentation algorithms to improve the validation and classification accuracy of the $\mathrm{CNN}$ model and achieved remarkable validation accuracy. Our classification method uses convolutional neural networks for classifying the images and early diagnosis of Pneumonia. Our findings yield an accuracy of $85.73 \%$, surpassing the previously top scoring accuracy of $78.73 \%$.

Keywords -Pneumonia, x-ray imaging, early diagnosis, deep learning, automation
\end{abstract}

\section{INTRODUCTION}

The risk of pneumonia is immense for many, especially in developing nations where billions face energy poverty and rely on polluting forms of energy. The WHO estimates that over 4 million premature deaths occur annually from household air pollution-related diseases including pneumonia .Over 150 million people get infected with pneumonia on an annual basis especially children under 5 years old . In such regions, the problem can be further aggravated due to the dearth of medical resources and personnel. For example, in Africa's 57 nations, a gap of 2.3 million doctors and nurses exists. For these populations, accurate and fast diagnosis means everything. It can guarantee timely access to treatment and save much needed time and money for those already experiencing poverty. Deep neural network models have conventionally been designed, and experiments were performed upon hem by human experts in a continuing trial-anderror method. This process demands enormous time, know-how, and resources. To overcome this problem, a novel but simple model is introduced to automatically perform optimal classification tasks with deep neural network architecture. The neural network architecture was specifically designed for pneu- monia image classification tasks. The proposed technique is based on the convolutional neural network algorithm, utilizing a set of neurons to convolve on a given image and extract relevant features from them. Demonstration of the efficacy of the proposed method with the minimization of the computational cost as the focal point was conducted and compared with the exiting stateof-the-art pneumonia classification networks. Pneumonia is an inflammatory response in the lung sacs called alveoli. Its often caused by bacteria, viruses, fungi and other microbes. As the germs reach the lung, white blood cells act against the germ and inflammation occurs in the sacs. Thus, alveoli get filled with pneumonia fluid and this fluid causes symptoms like coughing, trouble in breathing and fever. If the infection isnt acted upon during the early periods of the disease, pneumonia infection can spread throughout the body and result in the death of the individual, as a result of the inability to exchange gas in the lungs.

In recent times, $\mathrm{CNN}$-motivated deep learning algorithms have become the standard choice for medical image classifications although the state-of-the-art $\mathrm{CNN}$-based classification techniques pose similar fixated network ar- chitectures of the trial-and-error system which have been their designing principle. U-Net, SegNet, and Car- diacNet are some of the prominent architectures for medical image examination.

Models like evolutionary-based algorithms and reinforcement learning (RL) have been introduced to locate optimum network hyperparameters during training. However, these techniques are computationally expensive, gulping a ton of processing power. As an alternative, our study proposes a conceptually simple yet efficient network model to handle the pneumonia classification problem as shown in Figures 1 and 2. 


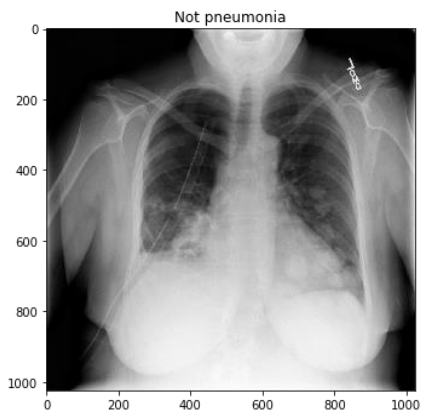

Fig.1

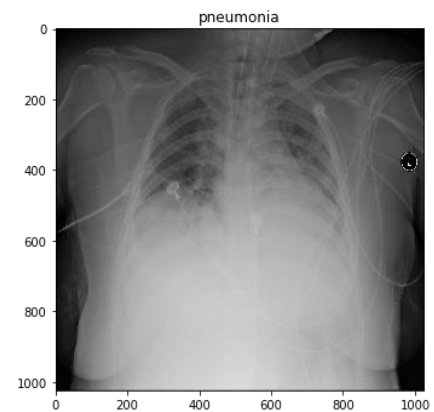

Fig.2

\section{RELATED WORK AND COMPARISON}

There has also been previous studies done on the early detection of pneumonia. Among the various other methods used by different studies, this paper focuses merely on Pneumonia and its classification.

The experimentation was conducted in Koc University Artificial Intelligence Laboratory, Istanbul, Turkey. In their study they presented a novel method for classifying pneumonia existence in an x-ray image. They proposed a two-step image processing before training our deep learning model, in order for making the features of an x-ray image clearer and explicit for easing the classification process. They, then, executed a convolutional neural network followed by a residual neural network for the classification process.

Their experimentation was conducted with similar means to ours. They used a 3 layer convolutional network for feature map acquisition for image preprocessing. In our experiment we use 3 convolutional layers, yielding a more efficient and a computationally less costly training process. Our preprocessing methods are similar to real life applications, unlike statistical means that might be ineffective when wide range of data is present. Our proposed architecture yields an accuracy of $85.73 \%$, while their study yielded an accuracy of $78.73 \%$.

\section{MATERIALS}

We present the detailed experiments and evaluation steps undertaken to test the effectiveness of the proposed model. Our experiments were based on a chest X-ray image dataset proposed in kaggle.com. We Used Keras open-source deep learning framework with tensorflow backend to build and train the convolutional neural network model. All experiments were run on a standard Laptop with an Nvidia GeForce GTX 1050Ti GPU card of 4 GB.

Dataset-The original dataset consists of three main folders (i.e., training, testing, and validation folders) and two subfolders containing pneumonia $(\mathrm{P})$ and normal $(\mathrm{N})$ chest X-ray images, respectively. A total of 5,856 X-ray images of anterior-posterior chests were carefully chosen from retrospective pediatric patients between 1 and 5 years old. The entire chest X-ray imaging was conducted as part of patients routine medical care. To balance the proportion of

data assigned to the training and validation set, the original data category was modified. We rearranged the entire data into training and validation set only. A total of 3,722 images

were allocated to the training set and 2,134 images were assigned to the validation set to improve validation accuracy.

\section{MODEL}

The model we build is a five layered convolutional network. The input image dimension is $(100,100,1)$. The input images are generated using ImageDataGenerator class and augmentation techniques are used in order to shearing, shifting and zooming in image to match the input dimension. The model is trained in batches of 32 images. The activation function used is ReLu (rectified linear unit) for hidden layer and sigmoid for output layer. The loss function used is binary crossentropy because of binary output. The optimizer function used in the model is adam (adaptive gradient descent with momentum) optimizer. The evaluation metric we used for model is accuracy with stands for percentage of images correctly classified to total images used.

\section{RESULT}

The accuracy curve is demonstrated in image below:

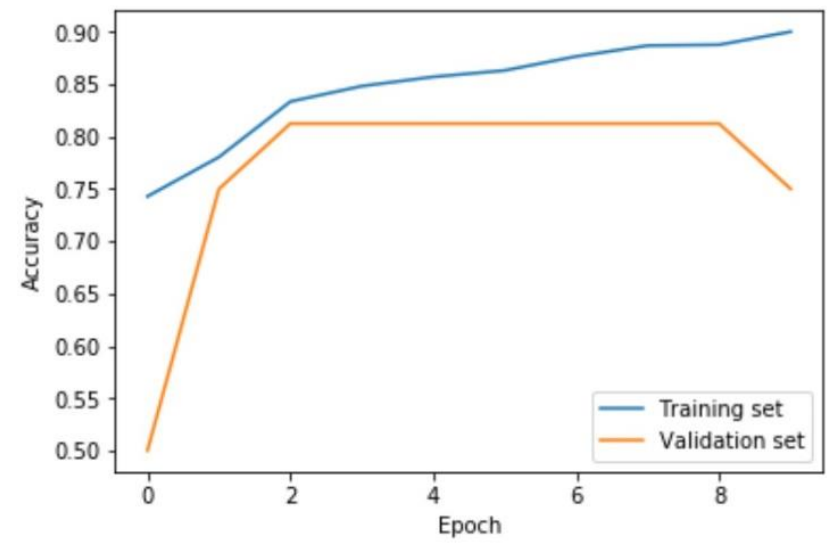

The Loss to Epoch graph is demonstrated in image as below:

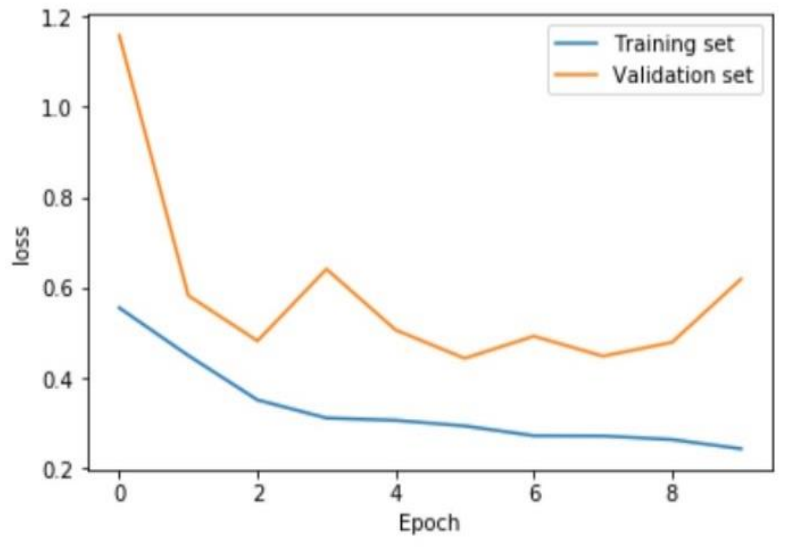


International Journal of Computer Applications Technology and Research

Volume 9-Issue 05, 183-185, 2020, ISSN:-2319-8656

\section{MATHEMATICAL DETAILS BEHIND WORKING OF CONVOLUTIONAL NEURAL NETWORKS}

$\mathrm{CNN}$ is primarily used to look for the patterns in an image. Feature selection in case of images which has hundreds and thousands of dimensions would be a painful process.

Feature selection happens all by itself during backpropagation over number of iterations. The input format of CNN is matrix ([[],[]]) and that of multilayer perceptron is tensor ([]).

Convolutional networks were inspired by biological processes in case of connectivity patterns of neurons. Unlike Multilayer Perceptron which uses fully connected layer, CNN uses different layers to detect patterns among images which are feed forwarded. Every image represent some pixels in simple terms.

We analyze the influence of nearby pixels on a particular pixel in an image by using a filter or a kernel.

Filters are tensors used track of spatial information. These filters learn to extract features like edge detection etc of objects in images in something called a convolutional layer. They help to filter out unnecessary or repetitive information during convolution operation.

There are multiple types of filters like high pass, low pass, gaussian etc which are used for different sort of operations. Then the convolution image matrix multiplies with filter matrix which is called Feature Map. Each filter is strided over the image using ot products between filter matrix and corresponding image pixel values. During the convolution operation size of image matrix decreases, if do not intend to do that then there is concept of padding. During padding redundant tensor of zero valued pixels is appended on edges of images, which preserve the size during convolutions. Next, we need to reduce the size of images, if they are too large. Pooling layers section would reduce the number of parameters when the images are too large.

Adding pooling layer then decrease the size of the image and hence decrease the complexity and computations.

Usually, an activation function ReLu is used in next layer. ReLU stands for Rectified Linear Unit for a non-linear operation.

The output is $f(\mathrm{x})=\max (0, \mathrm{x})$.

The purpose of ReLu is to add non-linearity to the convolutional network. In usual cases, the real-world data want our network to learn non-linear patterns which is the purpose of activation functions. The final step is to flatten our matrix and feed the values to fully connected layer.

We need to train the model in the same way, we train other neural networks. Using the certain number of epochs and then backpropagate to update weights and calculate the loss.

\section{CONCLUSION}

In this study, we present a novel method for classifying an $\mathrm{X}$-ray image on its possibility of exhibiting pneumonia in the early stags of the disease. We utilized a convolutional neural network approach for obtaining feature maps of the preprocessed X-ray images. Our method was convolutional neural network which yielded a high accuracy. Our most accurate experimentation model classifies the images with a $85.73 \%$ accuracy. Overall, we target the current drawback in medical diagnosis of pneumonia by the human high, and propose an alternative and more accurate way of diagnosing the disease with automation. Moreover, we target the limits caused by the gray scale of x-ray imaging, preventing the early diagnosis of the disease. Our study presents an efficient algorithm with a high performance for this classification task and can be improved through object detection algorithms for extracting the region with pneumonia.

\section{VIII.REFERENCES}

1. Deniz Yagmur Urey, Can Jozef Saul, and Can Doruk Taktakoglu Robert College of Istanbul, Istanbul, Turkey

Koc University Artificial Intelligence Laboratory, Istanbul, Turkey

Early Diagnosis of Pneumonia with Deep Learning.

2. He, K., Zhang, X., Ren, S., \& Sun, J. (2016). Deep residual learning for image recognition. In Proceedings of the IEEE conference on computer vision and pattern recognition (pp. 770778).

3. CheXNet:Radioogist-Level Pnemonia Detection on Chest -Ray with Deep Learning. https://arxiv.org/abs/1711.05225v3 